\title{
Solubilidade de compostos fenólicos e atividade antioxidante em extratos obtidos de resíduo de uva de vinho da Cultivar Bordô
}

No processo de produção de vinho o resíduo gerado corresponde em cerca de $20 \%$ da matéria-prima, que consiste de engaço, bagaço, sementes e borras ricas em compostos bioativos e valor nutricional que podem ser utilizados como complemento de ração animal ou para a adubação do solo. Visando dar um destino mais nobre para esse resíduo, objetivou-se investigar o efeito de solventes de extração em compostos fenólicos de resíduo de vinho da cultivar Bordô, bem como sua atividade antioxidante in vitro. Para tal finalidade, implantou-se um planejamento unifatorial - relação solvente e água - com dois solventes (metanol e etanol) combinados na proporção em volume de 0,50,75 e $100 \%$ de solvente com água, totalizando 8 tratamentos. As análises foram fenóis totais (FT) antocianinas totais (ANT), capacidade antioxidante (CA) e açúcares redutores (AR). Foram quantificados também o teor de ácido gálico, ácido cafeico e resveratrol por CLAE. Os resultados in vitro indicam que a mistura solvente e água, tanto usando etanol como metanol, na proporção de 50 ou $75 \%$, melhora a extração dos compostos fenólicos pelo aumento da solubilidade e, como consequência, facilita a permeabilidade e transferência desses compostos por difusão. Houve forte correlação direta entre a FT, CA e ANT, sendo que dos três compostos identificados por CLAE, o ácido cafeico destacou-se positivamente para atividade antioxidante. Entretanto, os resultados não são conclusivos com relação à natureza química que foi favorecida frente aos oito tratamentos in vitro, mas os resultados para AR indicam que estes estão presentes como compostos fenólicos complexos.

Palavras-chave: Extratos fenólicos; Capacidade antioxidante; Solubilidade; CLAE; in vitro.

\section{Solubility of phenolic compounds and antioxidant activity of extracts obtained from residue of wine grapes of the Bordô Cultivar}

Wine production generates large amounts of residues, accounting for around $20 \%$ of the raw material. Such residue consists of stem, bagasse, seeds, and lees, which are rich in bioactive compounds and nutritional value, being used to complement animal feed or in soil fertilization. In order to give a nobler destination to the residue, the objective of this study was to investigate the effect of extraction solvents on phenolic compounds from wine residue of the Bordô cultivar, as well as the in vitro antioxidant activity of the extracts. For this purpose, a factorial study design based on the solvent: water ratio was implemented with two solvents (methanol and ethanol) combined at $0,50,75$, and $100 \%$ proportion of solvent with water (v/v) for a total of eight treatments. Analyses of total phenols (TP), total anthocyanins (ANT), antioxidant capacity (AC), and reducing sugars (RS) were performed. Contents of gallic acid, caffeic acid, and resveratrol were also quantified through HPLC. The in vitro results indicate the blend of solvent and water with either ethanol or methanol at 50 or $75 \%$ improves the extraction of the phenolic compounds by increasing solubility and, consequently, facilitates the permeability and transfer of those compounds through diffusion. A strong direct correlation was found among TP, AC, and ANT, with caffeic acid standing out for its antioxidant activity among the three compounds identified through HPLC. However, the results are inconclusive regarding the chemical nature favored in the eight in vitro treatments, but the RS results indicate those compounds are present as complex phenolic compounds.

Keywords: Phenolic extracts; Antioxidant capacity; Solubility; HPLC, in vitro.

Caroline da Silva Rodrigues

Instituto Federal Sul-Rio-Grandense, Brasil

krol-17@hotmail.com

Izadora Oliveira Piegas (iD

Instituto Federal Sul-Rio-Grandense, Brasil

http://lattes.cnpq.br/1709436811493563

http://orcid.org/0000-0001-8808-287X

izadorapiegas@gmail.com

\section{Pâmela Moura Costa (iD)}

Instituto Federal Sul-Rio-Grandense, Brasil http://lattes.cnpq.br/9087350419603555 http://orcid.org/0000-0002-5540-8332 pamela.c.moura@hotmail.com
Bernardo dos Santos Vaz

Instituto Federal Sul-Rio-Grandense, Brasil http://lattes.cnpq.br/8068730182241990 bernardo@zebrafsh.com.br

Jander Luis Fernandes Monks (id)

Instituto Federal Sul-Rio-Grandense, Brasil

http://lattes.cnpq.br/71557455446456735 http://orcid.org/0000-0003-3616-3314 jandermonks@ifsul.edu.br

Ricardo Lemos Sainz (10

Instituto Federal Sul-Rio-Grandense, Brasil

http://lattes.cnpq.br/6460728200985124

http://orcid.org/0000-0001-9338-7993

ricardosainz@pelotas.ifsul.edu.br
Ricardo Peraça Toralles (it)

Instituto Federal Sul-Rio-Grandense, Brasil http://lattes.cnpq.br/9638201620937404 http://orcid.org/0000-0003-0898-1803 toralles@pelotas.ifsul.edu.br

Referencing this:

RODRIGUES, C. S.; PIEGAS, I. O.; COSTA, P. M.; VAZ, B. S.; MONKS, J. L. F.; SAINZ, R. L.; TORALLES, R. P.. Solubilidade de compostos fenólicos e atividade antioxidante em extratos obtidos de resíduo de uva de vinho da Cultivar Bordô. Revista Ibero Americana de Ciências Ambientais, v.12, n.5, p.106-120, 2021. DOI: http://doi.org/10.6008/CBPC2179$\underline{6858.2021 .005 .0010}$ 


\section{INTRODUÇÃO}

Em 2019 o Brasil produziu cerca de 1,4 milhões de toneladas de uvas. A região Sul é a maior produtora com quase $60 \%$ da produção nacional (IBGE, 2019). Estima-se que $20 \%$ do volume da matériaprima para a produção de vinhos e sucos de uvas seja o bagaço, parte não aproveitada e que a indústria na maioria das vezes trata como rejeito, desconsiderando o seu potencial para produção de subprodutos (MELLO, 2018). Diante deste cenário torna-se cada vez mais necessário a busca por alternativas adequadas para a destinação dos resíduos vitivinícolas, sendo que a inserção destes em outros produtos podem oferecer benefício à saúde, fato este que vem sendo ignorado (RIBEIRO, 2016; MELLO, 2018).

Para o Rio Grande do Sul o percentual citado é considerado muito expressivo, visto que aproximadamente 90 \% da produção de vinho nacional são realizadas nesse estado. Assim como o vinho, a produção de suco de uva também contribuiu para o aumento da geração de resíduo. Em 2018, por exemplo, somente por empresas do Rio Grande do Sul comercializaram-se cerca de 197 milhões de litros desse produto (MELLO, 2018).

Embora sejam citados na literatura trabalhos que discutem ou demonstram ser possível o reaproveitamento do resíduo da uva para diferentes fins, como a produção de cosméticos, alimentos funcionais, medicamentos (ZHU et al., 2015; GLAMPEDAKI et al., 2014) e fertilizantes (FERRER et al., 2001; ARVANITOYANNIS et al., 2008; CARMONA et al., 2012) é crescente o interesse pela avaliação do potencial antioxidante, antiproliferante e antilipidêmico, a partir da extração de compostos fenólicos presentes em extratos de semente, casca e ráquis de uva (KAUR et al., 2008; JIMÉNEZ et al., 2008; SHROTRIYA et al., 2015; SAHPAZIDOU et al., 2014, COLPO et al., 2019).

É crescente também a identificação e quantificação desses compostos (CAPONE et al., 2011; STALMACH et al., 2011). Por exemplo, Natividade et al. (2013), trabalhado com a identificação de 25 compostos por Cromatografia liquida de Alta Eficiência-CLAE em diferentes sucos de uva do vale São Francisco, identificou e quantificou 25 compostos fenólicos diferentes em sucos de uvas. Porém, ainda não está bem claro a bioatividade individual ou sinérgica desses compostos.

Em uvas e derivados tem-se encontrado diversos trabalhos que determinaram a atividade (ou capacidade) antioxidante em extratos in vitro (GONZÁLEZ-PARAMÁS et al., 2004; MAIER et al., 2009; ROCKENBACK et al., 2011; CHENG et al., 2012; FERNANDES et al., 2013; SOUZA et al., 2014; VERGARASALINAS et al., 2015; GONZÁLEZ-CENTENO et al., 2015). Já se encontra alguns trabalhos relacionando atividade antioxidante individual desses compostos. Por exemplo, Minussi et al. (2003), trabalhando com extratos de vinho branco e tinto, encontraram correlação forte entre o potencial antioxidante e os ácidos gálico e cafeico, mas fraca correlação entre o potencial antioxidante e o resveratrol.

Em relação aos estudos citados, cabe destacar que todos descreveram que utilizaram resíduos (semente e/ou cascas) obtidos a partir da produção de vinho. Com exceção de Rockenback et al. (2011) e de Souza et al. (2014), os quais utilizaram em seus respectivos experimentos o cultivar Isabel e Bordô - os demais autores, de uma maneira geral, avaliaram extratos de resíduos de uvas da espécie Vitis vinifera (Carbenet Sauvignon e Merlot, por exemplo). 
Ainda sobre os trabalhos mencionados, pode-se verificar que para a extração de compostos fenólicos do resíduo de uva foram, majoritariamente, avaliados: i) uma mistura de solventes, como metanol: água acidificado com HI (MAIER et al., 2009; ROCKENBACK et al., 2011; SAHPAZIDOU et al., 2014) ou etanol: água (MAIER et al., 2009; CHENG et al., 2012), ou ii) somente água (VERGARA-SALINAS et al., 2015; GONZÁLEZ-CENTENO et al., 2015). Em relação à composição de cada mistura, os autores apresentados estudaram diferentes relações, como 3:1 (etanol: água), 1:1 (metanol: água; etanol: água) e 90:9,5:0,5 (metanol: água: $\mathrm{HCl}$ ).

A solubilidade de compostos químicos orgânicos é um assunto de grande importância no ramo da química e com aplicação em diversas áreas tecnológicas, tais como: materiais, medicamentos e meio ambiente (MARTINS et al., 2013). A influência do solvente ou mistura de solventes na solubilidade é função do tipo predominante de ligação química, estrutura molecular, polaridade e tipos de interações intermoleculares. Tal fato é de suma importância para entender a operação de extração sólido-líquido e seu mecanismo transferência de massa (RODRÍGUEZ-JIMENES et al., 2013). Neste estudo, objetivou-se investigar o efeito de solventes de extração em compostos fenólicos de resíduo de vinho da cultivar Bordô, bem como sua atividade antioxidante in vitro.

\section{METODOLOGIA}

Os resíduos de vinho tinto da cultivar Bordô foram doadas pelo grupo de pesquisas VINHO NA TALHA - IFsul campus Pelotas, da safra 2018/2019. Os resíduos foram armazenados em congelador no laboratório de Bioquímica do IFSul campus pelotas até o momento da realização das análises.

As amostras foram congeladas a $-80^{\circ} \mathrm{C}$ em ultra freezer INDREL ${ }^{\circledR}$ IULT $335 \mathrm{D}$ e liofilizadas em liofilizador de bancada (Modelo L101 LIOTOP) durante 24 horas. Após este processo as amostras foram trituradas e tamisadas em peneiras de 20 mesh. Em seguida, foram armazenados em frascos âmbar a $-20^{\circ} \mathrm{C}$ até o momento das análises. Após, as amostras foram secas em estufa a $80{ }^{\circ} \mathrm{C}(515-\mathrm{C}$, Fanen) por 10 minutos para inativar as enzimas responsáveis pelo escurecimento enzimático. Deixou-se resfriar em dessecador, seguido por desidratação em estufa com circulação de ar (Modelo TE-394/2, Tecnal ${ }^{\circledR}$, Piracicaba, Brasil) a $50^{\circ} \mathrm{C}$ durante 24 horas. A espessura da amostra foi de $0,5 \mathrm{~cm}$ a velocidade do ar de 0,78 $\mathrm{m} \mathrm{s}^{-1}$. Após a desidratação as amostras foram trituradas em moinho de facas (QUIMIS Modelo Q298A21) e tamisadas em peneiras de 20 mesh. Em seguida, foram armazenadas em frascos âmbar a $-20{ }^{\circ} \mathrm{C}$ até o momento das análises. Os resíduos desidratados e moídos com circulação de ar apresentaram aproximadamente $8 \%$ de umidade em úmida e doravante foram chamados de 'resíduo-DM'.

\section{Efeito da relação solvente e água na extração in vitro}

Para analisar o efeito da relação solvente e água no teor de fenóis totais, antocianinas, capacidade antioxidante e teor de açúcares redutores, um planejamento unifatorial - relação solvente e água - com dois solventes (metanol e etanol) combinados na proporção em volume de 0, 50, 75 e $100 \%$ de solvente com água, totalizando 8 tratamentos (Tabela 1). Cada tratamento foi feito em triplica da unidade 
experimental (resíduo-DM), sendo que os tratamentos 1 e 5 são tratamentos iguais usados como controle de ocorrência do erro do tipo I. Em um béquer, juntou-se 5,000 gramas de resíduo-DM de vinho Bordô e $50,0 \mathrm{~mL}$ da mistura de solventes como descrito na Tabela 1. A mistura de solvente foi previamente acidificada a pH 1,5. O conteúdo do béquer foi transferido para um frasco de erlenmeyer e conduzido em agitação orbital (Quimis-Q816M22) a $50 \mathrm{rpm}, 30{ }^{\circ} \mathrm{C}$ durante 2 horas. Posteriormente, foi centrifugado por 10 minutos e $3200 \mathrm{rpm}$. O extrato obtido de resíduo desidratado e moído foi doravante chamado de 'extrato-RDM' e foram armazenados em frascos Falcon a $-20^{\circ} \mathrm{C}$ até o momento das análises para fenóis totais, antocianinas, capacidade antioxidante e análise cromatográfica CLAE.

Tabela 1: Planejamento experimental para o sistema solvente: água.

\begin{tabular}{|l|l|l|}
\hline Tratamento & Solventes & $\% \mathrm{v} / \mathrm{v}$ \\
\hline 1 & Metanol e água & 0 \\
\hline 2 & Metanol e água & 50 \\
\hline 3 & Metanol e água & 75 \\
\hline 4 & Metanol e água & 100 \\
\hline 5 & Etanol e água & 0 \\
\hline 6 & Etanol e água & 50 \\
\hline 7 & Etanol e água & 75 \\
\hline 8 & Etanol e água & 100 \\
\hline
\end{tabular}

\section{Fenóis totais}

O teor de fenóis totais (TFT) foi determinado segundo a metodologia adaptada de Singleton et al. (1965), com determinação colorimétrica utilizando o reagente Folin-Ciocalteau a $725 \mathrm{~nm}$. Para cada extrato uma alíquota de $1 \mathrm{~mL}$ foi recolhida em um balão volumétrico de $100 \mathrm{~mL}$, no qual se adicionou $60 \mathrm{~mL}$ de água deionizada e $5 \mathrm{~mL}$ de Folin-Ciocaulteau. Após, deixou-se o meio reacional em repouso por 8 minutos e $20 \mathrm{~mL}$ de carbonato de sódio a $20 \%$ p/v foi adicionado. Completou-se o volume com água deionizada. Após 2 horas, uma alíquota do meio reacional foi retirada para medir a absorbância em 725 nm, utilizando um espectrofotômetro AJX-1000 UV/VIS. A concentração de fenóis totais foi determinada através da Equação 1: onde A é absorbância, MM é massa molecular do ácido gálico $(188,14 \mathrm{~g} / \mathrm{mol}), \varepsilon$ é absortividade molar e C é o caminho ótico $1 \mathrm{~cm}$. Os resultados expressos em $\mathrm{mg}$ de ácido gálico por litro de extrato, através da construção de uma curva de calibração para ácido gálico.

\section{Antocianinas}

$$
F T=\frac{A * M M * 10^{5}}{\varepsilon * C}
$$

O teor de antocianinas do extrato foi determinado pelo método de Lees et al. (1972). Para cada extrato uma alíquota de $1 \mathrm{~mL}$ foi recolhida em um béquer de $100 \mathrm{~mL}$, no qual se adicionou 25,0 mL de etanol pH de 1,0. A mistura permaneceu sob agitação por 1 hora e logo após, filtrou-se com papel 
Whatman $\mathrm{n}^{\circ} 26$ e transferido para balão volumétrico de $50,0 \mathrm{~mL}$, completando o volume com etanol pH 1,0. Uma alíquota da mistura foi retirada para medida da absorbância em $520 \mathrm{~nm}$, utilizando um espectrofotômetro AJX-1000 UV/VIS. A concentração de antocianinas totais foi determinada através da Equação 2: onde A é a absorbância e FD é o fator de diluição. Os resultados expressos em $\mathrm{mg} \cdot 100 \mathrm{~g}^{-1}$.

\section{Capacidade antioxidante}

$$
A T=\frac{A x F D}{98,2}
$$

A capacidade antioxidante relativa foi determinada segundo o método de OZGEN (2006) em termos de capacidade antioxidante equivalente ao Trolox (TEAC) em 2,2-difenil-1-picrilhidrasil (DPPH). Alíquotas de $20 \mu \mathrm{l}$ dos extratos foram adicionados a $3 \mathrm{~mL}$ da solução do radical livre DPPH a $100 \mu \mathrm{M}$. Após 30 minutos, fez-se a leitura da absorbância a $515 \mathrm{~nm}$ para DPPH, utilizando um espectrofotômetro AJX-1000 UV/VIS. Os valores encontrados foram calculados de acordo com a Equação 3: onde $A_{E}$ é a absorbância do branco, $A_{A}$ é a absorbância da amostra, $\mathrm{V}$ é o volume de centrifugado em $\mathrm{mL}, \mathrm{m}$ é a massa de amostra em $\mathrm{g}$, $\varepsilon$ é absortividade molar do Trolox em DPPH e FD é o fator de diluição. Os resultados expressos em mg por litro de extrato, através da construção de uma curva padrão Trolox em DPPH.

\section{Açúcares redutores}

$$
T E A C=\frac{\left(A_{E}-A_{A}\right)}{\varepsilon} \times \frac{V}{m} \times F D
$$

Os açúcares redutores foram determinados pelo método colorimétrico da glicose usando $1 \mathrm{~mL}$ 3,5dinitrosalicílico (DNS, Sigma Aldrich Chemie $\mathrm{GmbH}$, Alemanha), $1 \mathrm{~mL}$ de extrato e $1 \mathrm{ml}$ de água, seguido de banho-maria fervente (JProlab Modelo 8370) por 5 minutos. No branco, foi utilizada água destilada no lugar do meio de cultura. Todas as medidas foram conduzidas em triplicatas. A transmitância resultante dessa reação foi medida a $490 \mathrm{~nm}$, utilizando um espectrofotômetro AJX-1000 UV/VIS. A massa de glicose no meio reativo, foi determinada através da curva padrão de glicose Equação 4: onde $A B$ é a absorbância do branco, AA é a absorbância da amostra, A é a constante de proporcionalidade de 1,72274; V é o volume final do meio reativo, em mL, e F é o fator de diluição. Os limites de detecção e quantificação são iguais a $32,5 \mathrm{mg} \mathrm{L}^{-1}$ e $108,44 \mathrm{mg} \mathrm{L}^{-1}$ respectivamente (FERREIRA, 2016).

$$
m=\frac{(A A-A B)}{A} V \cdot F
$$

\section{Análise por CLAE-DAD}

A análise de Cromatografia Líquida de Alta Eficiência foi realizada em um cromatógrafo da marca Agilent Technologies, detector de arranjos de diodos (DAD), com varredura entre 140-800 nm, usando uma coluna de separação analítica Zorbax $C_{18}(3,9 \mathrm{~cm} \times 150 \mathrm{~mm} \times 4 \mu \mathrm{m})$. O eluente de escolha foi acetonitrila Merck grau HPLC e água ultrapura (20:80) ajustando-se o pH 2,5 com ácido acético p.a Merck. A injeção da amostra foi de $20 \mu \mathrm{L}$ a $35{ }^{\circ} \mathrm{C}$ com fluxo de $0,5 \mathrm{~mL} \mathrm{~min}^{-1}$. A quantificação foi feita por padronização externa, 
com os padrões gálico, cafeico e resveratrol da marca Sigma, usando curva de calibração (Tabela 8 -

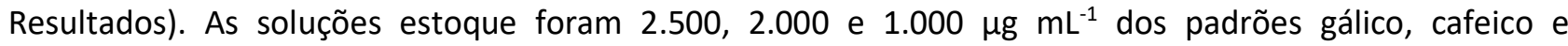
resveratrol, respectivamente. Os compostos fenólicos foram identificados comparando os tempos de retenção dos padrões e através de informações obtidas dos espectros de absorção no UV-visível em comprimento de onda de 250 a $320 \mathrm{~nm}$. O teor dos compostos fenólicos foi expresso em $\mu \mathrm{g} \mathrm{mL}{ }^{-1}$ de extrato.

\section{Análise estatística}

O software Statistica 13.4 (STATSOFT EUA) foi utilizado para calcular os coeficientes de regressão linear, o coeficiente de determinação e a análise de variância (ANOVA), bem como para gerar os gráficos em duas e três dimensões. Os intervalos de confiança dos coeficientes foram calculados multiplicando-se o erro padrão por Student-t $(n-2)$, ajustado aos graus de liberdade $(p=0,05)$. A comparação de médias foi feita pelo teste de Tukey.

\section{RESULTADOS E DISCUSSÃO}

\section{Curva de calibração para determinação de fenóis totais}

Na Figura 1 tem-se uma curva de calibração típica para determinação de fenóis totais com coeficiente de determinação $\left(R^{2}=0,9815\right)$. A constante de proporcionalidade $(A)$ entre a absorbância e a concentração de fenóis totais e coeficiente linear da reta (B) foram calculadas por regressão linear. O valor de A (coeficiente angular) foi significativo $(p$ [20,01) e com intervalo de confidência de $0,001445 \pm 0,000198 \mathrm{~L}$ $\mathrm{mg}^{-1} \mathrm{~cm}^{-1}$. O valor de B é 0,007511 $\pm 0,052207 \mathrm{mg} \mathrm{L}^{-1}$. Os Limites de Detecção (LD) e Quantificação (LQ) iguais a 76,69204 e $255,6401 \mathrm{mg} \mathrm{L}^{-1}$. A absortividade molar para fenóis foi de $27280 \mathrm{~L} \mathrm{~mol}^{-1} \mathrm{~cm}^{-1}$. SILVA et al. (2011), usando o mesmo método de Singleton et al. (1965), obteve uma curva semelhante com uma absortividade molar igual a $20.500 \mathrm{~L} \mathrm{~mol}^{-1} \mathrm{~cm}^{-1}$.

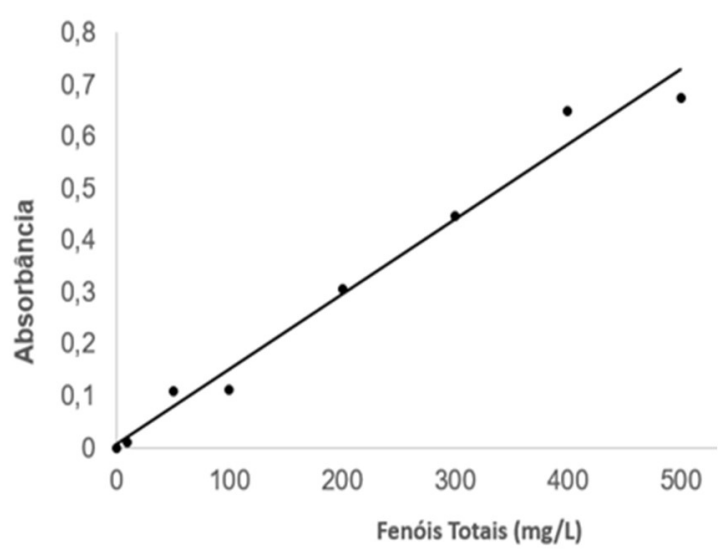

Figura 1: Curva de calibração para fenóis totais.

A análise de variância (ANOVA) da curva de calibração para fenóis totais é mostrada na Tabela 2.

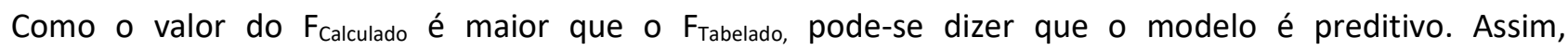
considerando que o coeficiente linear não foi significativo, pode-se afirmar que a curva segue a Lei de 
Lambert-Beer e pode ser calculado pela Equação 1 descrita na Metodologia.

Tabela 2: Análise de variância da curva de calibração para fenóis totais

\begin{tabular}{|l|l|l|l|l|l|}
\hline Efeito & S.Q & G.L & M.Q & F Caculado \\
\hline Regressão & 0,518686 & 1 & 0,518686 & 317,6414 & \\
\hline Resíduo & 0,009798 & 6 & 0,001633 & & \\
\hline Total & 0,528483 & & & & \\
\hline
\end{tabular}

$\mathrm{F}(1,6)$ tabelado $=13,75$ a $1 \%$

\section{Curva de calibração para capacidade antioxidante}

A curva de calibração para capacidade antioxidante encontra-se na Figura 2. A constante de proporcionalidade (A) entre a absorbância e a concentração de fenóis e o coeficiente linear da reta (B) também foram calculadas por regressão linear. $O$ valor de $A$ (coeficiente angular) foi significativo $(p[0,01)$ e com intervalo de confidência de $0,00074 \pm 0,00007$. O valor de $B$ é $-0,01084 \pm 0,03938$. Os Limites de Deteç̧ão (LD) e Quantificação (LQ) iguais a 69,692 e 232,307 mg L-1 (Tabela 5). A absortividade molar para capacidade antioxidante foi de $186 \mathrm{~L} \mathrm{~mol}^{-1} \mathrm{~cm}^{-1}$. Silva et al. (2011), usando o mesmo método de Ozgen (2006), obteve uma curva semelhante com uma absortividade molar igual a $188 \mathrm{~L} \mathrm{~mol}^{-1} \mathrm{~cm}^{-1}$.

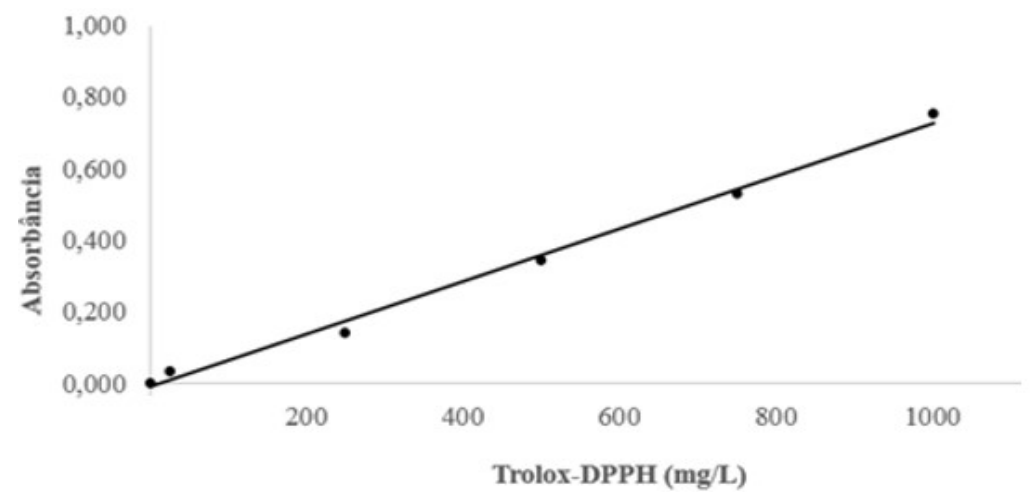

Figura 2: Curva de calibração para capacidade antioxidante

A análise de variância (ANOVA) da curva de calibração para capacidade antioxidante é mostrada na

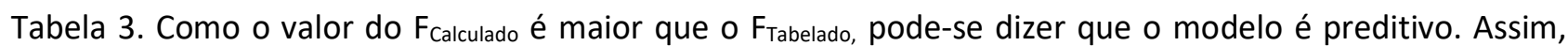
considerando que o coeficiente linear não foi significativo, pode-se afirmar que essa também segue a Lei de Lambert-Beer e pode ser calculado pela Equação 3.

Tabela 3: Análise de variância da curva de calibração para capacidade antioxidante.

\begin{tabular}{|l|l|l|l|l|}
\hline Efeito & S.Q & G.L & M.Q & Fcaculado \\
\hline Regressão & 0,443038 & 1 & 0,443038 & 581,1595 \\
\hline Resíduo & 0,003049 & 4 & 0,000762 & \\
\hline Total & 0,446087 & & & \\
\hline
\end{tabular}

$\mathrm{F}(1,4)$ tabelado $=21,20$ a $1 \%$ 


\section{Efeito da relação solvente e água na extração in vitro}

Na Tabela 4 encontram-se listados os resultados para os teores de antocianinas, fenóis totais, capacidade antioxidante relativa (TEAC-DPPH) e açúcares redutores para o efeito da relação solvente e água na extração in vitro. Para fenóis totais, os ensaios $2\left(1.000,259 \mathrm{mg} \mathrm{GAE} \mathrm{L}{ }^{-1}\right), 3\left(1.085,603 \mathrm{mg} \mathrm{GAE} \mathrm{L}^{-1}\right)$, $4\left(895,201 \mathrm{mg} \mathrm{GAE} \mathrm{L}^{-1}\right), 6\left(1.016,602 \mathrm{mg} \mathrm{GAE} \mathrm{L}^{-1}\right)$ e $7\left(953,567 \mathrm{mg} \mathrm{GAE} \mathrm{L}^{-1}\right)$ foram significativamente superiores aos demais tratamentos $(p \leq 0,05)$. Também se observou esse comportamento para ANT e CA (Tabela 4). Tal fato indica que a mistura dos solventes em água, tanto $50 \%$ em volume como $75 \%$ volume, melhorou a solubilidade dos compostos fenólicos, independente do solvente na mistura ser metanol ou etanol. Cheng et al. (2012), comparando três misturas solvente e água a $50 \%$ (v/v), na extração de compostos fenólicos de resíduo de uva das cultivares pinot noir (PN) e pinot meunir (PM) na temperatura ambiente, encontraram para PN 148,4 mg GAE por grama extrato e para PM 77,5 mg GAE por grama extrato, ambos os valores para mistura metanol e água $50 \%$, que foi superior tanto mistura etanol e água como a mistura acetona e água a 50 \%. Para capacidade antioxidante observaram o mesmo comportamento, mas não mediram antocianinas totais. Outros autores têm demonstrado a importância da relação solvente água na extração, bem como o tipo de solvente (BABBAR et al., 2011; NEGRO et al., 2003; VENKATESAN et al., 2019).

Tabela 4: Fenóis totais (FT), antocianinas totais (ANT) e capacidade antioxidante (CA) e açúcares redutores que foram obtidos usando diferentes relações solvente e água $25^{\circ} \mathrm{C}$.

\begin{tabular}{|l|l|l|l|l|l|l|}
\hline Solventes & Tratamento & Proporções & $\begin{array}{l}\text { FT } \\
\left(\mu \mathbf{G A E ~ g}^{-1}\right)\end{array}$ & $\begin{array}{l}\text { ANT } \\
\left(\mathbf{m g ~ 1 0 0 ~ g}^{-1}\right)\end{array}$ & $\begin{array}{l}\text { CA } \\
\left(\mathbf{m g ~ L}^{-1}\right)\end{array}$ & $\begin{array}{l}\text { AR } \\
\left(\mathbf{g ~ L}^{-1}\right)\end{array}$ \\
\hline Metanol & 1 & 0 & $385,473 \mathrm{~b}$ & $137,8140 \mathrm{~b}$ & $382,381 \mathrm{~b}$ & $1,06 \mathrm{~b}$ \\
\hline Metanol & 2 & 50 & $1.000,259 \mathrm{a}$ & $533,2654 \mathrm{a}$ & $989,048 \mathrm{a}$ & $2,13 \mathrm{a}$ \\
\hline Metanol & 3 & 75 & $1.085,603 \mathrm{a}$ & $603,1908 \mathrm{a}$ & $1.140,476 \mathrm{a}$ & $2,02 \mathrm{a}$ \\
\hline Metanol & 4 & 100 & $895,201 \mathrm{a}$ & $529,1921 \mathrm{a}$ & $957,619 \mathrm{a}$ & $0,47 \mathrm{~b}$ \\
\hline Etanol & 5 & 0 & $316,991 \mathrm{~b}$ & $100,8147 \mathrm{~b}$ & $288,095 \mathrm{~b}$ & $1,44 \mathrm{~b}$ \\
\hline Etanol & 6 & 50 & $1.016,602 \mathrm{a}$ & $422,9464 \mathrm{a}$ & $880,952 \mathrm{a}$ & $2,28 \mathrm{a}$ \\
\hline Etanol & 7 & 75 & $953,567 \mathrm{a}$ & $448,7441 \mathrm{a}$ & $857,143 \mathrm{a}$ & $2,28 \mathrm{a}$ \\
\hline Etanol & 8 & 100 & $309,209 \mathrm{~b}$ & $118,4657 \mathrm{~b}$ & $402,619 \mathrm{~b}$ & $2,15 \mathrm{a}$ \\
\hline
\end{tabular}

*Médias seguidas pelas mesmas letras não diferem entre si pelo Teste de Tukey ( $p \leq 0,05)$. Os coeficientes de variação experimental ficaram entre 10 e $20 \%$. Os oito tratamentos foram repetidos em triplicata.

Por outro lado, os tratamentos 1 e 5, somente água como solvente de extração, usados como controle do erro do tipo I, não apresentaram diferença entre si por Tukey $(p \leq 0,05)$ e tiveram resultados inferiores para FT, ANT e CA in vitro (Tabela 4). O tratamento 8, somente etanol como solvente de extração, também inferior para FT, ANT e CA. Bucić-kojić et al. (2009), estudando a influência da temperatura e da mistura etanol e água na extração de compostos fenólicos de semente de uva da cultivar Frankovka, também observaram os menores valores para FT para água pura (FT= $30.87 \mathrm{mg} \mathrm{g}^{-1}$ ), seguido de etanol puro ( $\left.\mathrm{FT}=94,44 \mathrm{mg} \mathrm{g}^{-1}\right)$. Assim como neste trabalho, os referidos autores encontraram maior teor de fenóis totais para mistura $50 \%$ em volume de etanol, porém eles trabalharam a $80{ }^{\circ} \mathrm{C}$, FT igual 129.59 
$\mathrm{mg} \mathrm{g}^{-1}$. Para antocianidinas e atividade antioxidante encontraram maior teor para $50 \%$ em volume de etanol. Quanto ao efeito da temperatura, para mistura $50 \%$ etanol e água, o valor de $\mathrm{FT}$ a $80{ }^{\circ} \mathrm{C}$ praticamente dobrou quando comparado com a temperatura $25{ }^{\circ} \mathrm{C}\left(\mathrm{FT}=70,77 \mathrm{mg} \mathrm{g}^{-1}\right)$.

Para teor de açúcares redutores, observou-se comportamento semelhante aos resultados encontrados para FT, ANT e CA, ou seja, os tratamentos $2\left(2,13 \mathrm{~g} \mathrm{~L}^{-1}\right), 3\left(2,02 \mathrm{~g} \mathrm{~L}^{-1}\right), 6\left(2,28 \mathrm{~g} \mathrm{~L}^{-1}\right)$ e $7(2,28 \mathrm{~g}$ $\left.L^{-1}\right)$ foram significativamente superior aos demais $(p \leq 0,05)$. A exceção foi o tratamento $4,100 \%$ de metanol, que foi significativamente superior para FT, ANT e CA, mas não para o AR. O tratamento 8, $100 \%$ etanol, ficou no mesmo nível de significância dos tratamentos 2, 3, 6 e 7. Por outro lado, observou-se um baixo teor de AR nos tratamentos 1 e 5, que é $100 \%$ água. Isto provavelmente ocorreu porque parte dos açúcares redutores está combinado com as antocianidinas para formar a antocianina mesmo após a extração a temperatura ambiente. Normalmente, a glicose livre é mais solúvel em água do que em etanol e suas misturas (ALVES et al., 2007). Por outro lado, as antocianidinas sozinhas são pouco solúveis em água e bastante solúvel em etanol e suas misturas, observado nos estudos de Bucić-Kojić et al. (2009). Assim, considerando que parte dos açúcares redutores estão combinados na forma de antocianinas, os dois efeitos estão contribuindo no valor de AR no tratamento 8, somente etanol.

Segundo Jayaprakasha et al. (2001), a presença de água melhora a permeabilidade do tecido celular e, portanto, permite uma melhor massa de transferência por difusão, porém muitos autores relatam que a água, quando utilizada como único solvente, indica resultados inferiores para FT, ANT e CA (CACACE et al., 2003; BUCIĆ-KOJIĆ et al., 2009; ĆUJIĆ et al., 2016). Além do provável efeito sinérgico ainda não bem documentado na literatura, a melhor explicação para esse fato é que a água recupera os compostos bioativos solúveis em água na miríade de compostos fenólicos presentes em tecidos vegetais. Outro fato importante é se o composto fenólico encontra-se livre ou não no tecido vegetal ou após a extração. Por exemplo, Lou et al. (2014) encontraram para o composto p-cumárico FT de $355 \mathrm{mg}$ GAE $100 \mathrm{~g}^{-1}$, após um pré-aquecimento antes da extração, sendo que o p-cumárico na forma solúvel contribuiu com $24,2 \%$ do valor total de FT e o p-cumárico na forma insolúvel 63,8 \%. A forma solúvel foi testada em água quente e acetato de etila como solventes extratores.

\section{Correlação entre FT e ANT com capacidade antioxidante}

Os coeficientes de correlação calculados para fenóis totais versus capacidade antioxidante e antocianinas totais versus capacidade antioxidante para os oitos tratamentos in vitro são mostrados nas Figuras 3 e 4. Tanto FT ( $r=0,95496)$ como ANT $(r=0,98455)$ mantiveram uma correlação forte e positiva com CA. Resultados semelhantes foram encontrados por Cheng et al. (2012) para resíduo de uva. Outros autores têm demonstrado essa correlação para outras frutas, vegetais e seus resíduos (CANTILLANO et al., 2012; XU et al., 2009; PASKO et al., 2009). 


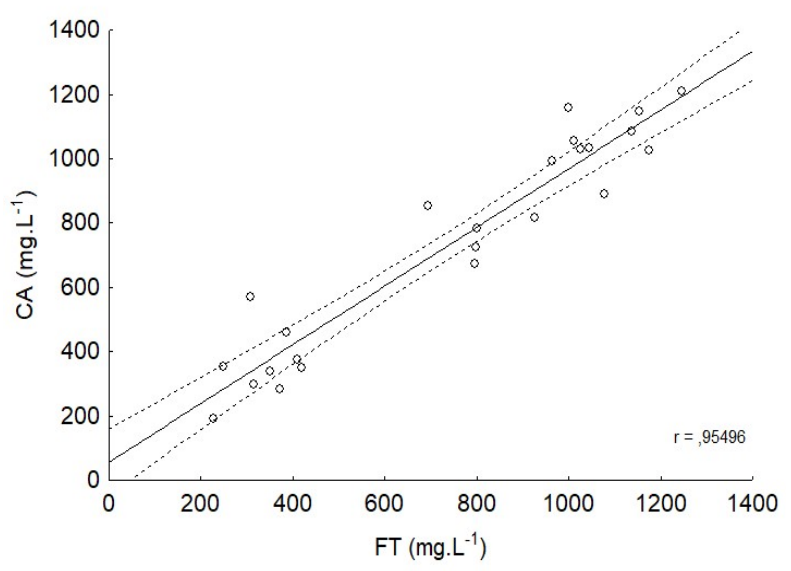

Figura 3: Correlação entre CA e FT para os extratos in vitro.

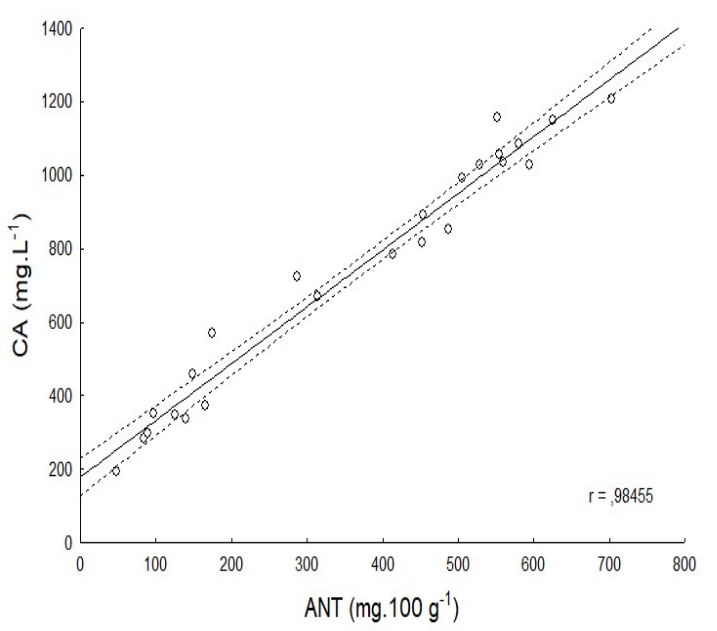

Figura 4: Correlação entre CA e ANT para os extratos in vitro.

\section{Quantificação de ácido gálico, ácido cafeico e resveratrol por CLAE}

Na Tabela 5 tem-se os parâmetros, os limites de detecção, quantificação e a análise estatística das curvas de calibração do ácido gálico, ácido cafeico e resveratrol. Todas mostraram-se preditivas decorrente de $F_{\text {calculado }}$ bem superior ao $F_{\text {tabelado. }}$.

Tabela 5: Parâmetros das curvas de calibração para ácido gálico, ácido cafeico e resveratrol.

\begin{tabular}{|l|l|l|l|}
\hline Coeficientes & \multicolumn{2}{l}{} & \multicolumn{2}{l|}{ Valores ${ }^{\text {a }}$} \\
\hline & $\begin{array}{l}\text { Ácido gálico } \\
\left(\mu \mathrm{g} \cdot \mathrm{mL}^{-1}\right)\end{array}$ & $\begin{array}{l}\text { Ácido cafeico } \\
\left(\mu \mathrm{g} \cdot \mathrm{mL}^{-1}\right)\end{array}$ & $\begin{array}{l}\text { Resveratrol } \\
\left(\mu \mathrm{g} \cdot \mathrm{mL}^{-1}\right)\end{array}$ \\
\hline A (angular) & $100,8 \pm 116,4^{* *}$ & $90,1 \pm 1,35^{* *}$ & $179,7 \pm 3.70^{* *}$ \\
\hline B (linear) & $-388,1 \pm 3,83^{* *}$ & $48,6 \pm 41,0^{* *}$ & $69,0 \pm 112,1^{\mathrm{Ns}}$ \\
\hline $\mathrm{R}^{2}$ & 0,98 & 0,99 & 0,99 \\
\hline LD & 4,04 & 1,59 & 2,18 \\
\hline LQ & 12,2 & 4,82 & 6,61 \\
\hline F calculado & 3046,7 & 19620,21 & 10433,28 \\
\hline F tabelado (1,16) & 8,53 & 8,53 & 8,53 \\
\hline
\end{tabular}

${ }^{a}$ valor ? intervalo de confidência a $\mathrm{p}=0,05 .{ }^{* *}$ Significativo ( $p$ ??0,01). ${ }^{\text {ns }}$ Não significativo.

O espectro cromatograma obtido para soluções de ácido gálico, ácido caféico e resveratrol e seus 
respectivos tempos de retenção estão na Figura 5. Como podemos ver, considerando as características da coluna $\mathrm{C} 18$, o ácido gálico é o mais polar e a ordem de polaridade é ácido gálico > ácido cafeico > resveratrol.

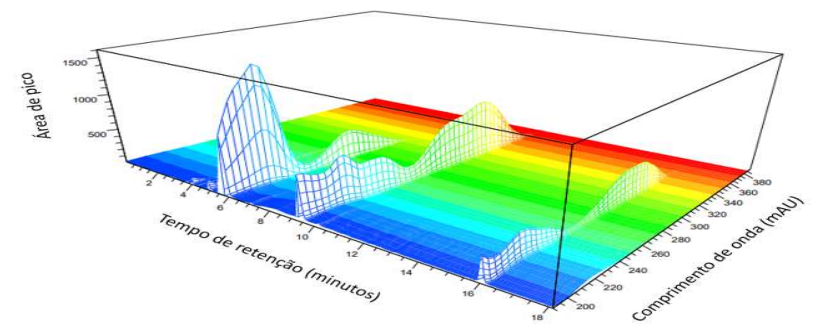

Figura 5: Espectro cromatograma dos compostos fenólico e seus respectivos tempos de retenção (TR): ácido gálico ( $T R=6,012$ minutos, $200 \mathrm{~nm}$ ); ácido cafeico ( $T R=8,468$ minutos, $320 \mathrm{~nm}$ ) e resveratrol $(16,015$ minutos, $320 \mathrm{~nm})$.

Na Tabela 6 tem-se o estudo do efeito da relação solvente e água na extração in vitro do ácido gálico, ácido cafeico e resveratrol determinados por CLAE. Para o ácido gálico, os tratamentos 1 (30,3 $\mu \mathrm{g}$ $\left.\mathrm{mL}^{-1}\right)$ e $5\left(38,3 \mu \mathrm{g} \mathrm{mL}^{-1}\right)$, somente água como solvente, foram significativamente superiores aos demais tratamentos $(p \leq 0,05)$. Lou et al. (2014) demonstraram a boa solubilidade desse ácido em água e acetato de etila.

Os tratamentos $3\left(37,8 \mu \mathrm{g} \mathrm{mL}^{-1}\right)$ e $4\left(37,3 \mu \mathrm{g} \mathrm{mL}^{-1}\right)$ foram significativamente superiores para ácido cafeico $(\mathrm{p} \leq 0,05)$, seguido dos tratamentos $2\left(29,5 \mu \mathrm{g} \mathrm{mL}^{-1}\right), 6(27,2 \mu \mathrm{g} \mathrm{mL}-1)$ e $7\left(29,10 \mu \mathrm{gL}^{-1}\right)$. Tal fato indica que o ácido cafeico teve um comportamento semelhante aos resultados para FT, ANT e CA, ou seja, os maiores valores absolutos para ácido cafeico foram encontrados para as misturas 50 e $75 \% \mathrm{v} / \mathrm{v}$, tanto para metanol como etanol, sendo que o metanol puro se mostrou melhor que o etanol puro e água pura para extração de ácido cafeico. Para extrato (75\% v/v de etanol) de resíduo prensado semente de uva cultivar Lemberger, Maier et al. 2009 encontraram 127,7 mg kg-1 de ácido gálico e 12,3 mg Kg-1 ácido cafeico.

Tabela 6: Ácido gálico, ácido cafeico, resveratrol que foram obtidos usando diferentes relações solvente e água a 25 ${ }^{0} \mathrm{C}$.

\begin{tabular}{|c|c|c|c|c|c|}
\hline Solventes & Tratamento & Proporções & $\begin{array}{l}\text { Ácido gálico } \\
\text { ( } \mu \mathrm{g} \mathrm{mL}^{-1} \text { ) }\end{array}$ & $\begin{array}{l}\text { Ácido cafeico } \\
\left(\mu \mathrm{g} \mathrm{mL}^{-1}\right)\end{array}$ & $\begin{array}{l}\text { Resveratrol } \\
\left(\mu \mathrm{g} \mathrm{mL}^{-1}\right)\end{array}$ \\
\hline Metanol & 1 & 0 & $30,3 \mathrm{~b}$ & $1,88 \mathrm{~d}$ & $0,0 \mathrm{e}$ \\
\hline Metanol & 2 & 50 & $19,6 \mathrm{c}$ & $29,5 \mathrm{~b}$ & $0,0 \mathrm{e}$ \\
\hline Metanol & 3 & 75 & $13,7 \mathrm{~d}$ & $37,8 \mathrm{a}$ & $0,189 \mathrm{c}$ \\
\hline Metanol & 4 & 100 & $0,0 \mathrm{e}$ & 37,3 a & $0,289 a$ \\
\hline Etanol & 5 & 0 & $38,3 \mathrm{a}$ & $2,04 \mathrm{~d}$ & $0,0 \mathrm{e}$ \\
\hline Etanol & 6 & 50 & $0,0 \mathrm{e}$ & $27,2 \mathrm{~b}$ & $0,0 \mathrm{e}$ \\
\hline Etanol & 7 & 75 & $0,0 \mathrm{e}$ & $29,1 b$ & $0,234 \mathrm{~b}$ \\
\hline Etanol & 8 & 100 & $0,0 \mathrm{e}$ & $5,34 \mathrm{c}$ & $0,133 d$ \\
\hline
\end{tabular}

*Médias seguidas pelas mesmas letras não diferem entre si pelo Teste de Tukey $(p \leq 0,05)$. Coeficientes de variação experimental ficaram entre 10 e $20 \%$. Todos menores que LD para o teor de resveratrol. Os oito tratamentos foram repetidos em triplicata.

Todos os valores encontrados para resveratrol ficaram abaixo do limite de detecção LD, que é a 
mais baixa concentração de analito que pode ser detectado de forma confiável e distinto de zero; porém o tratamento 4, somente metanol, foi onde observou-se o maior valor para resveratrol. Natividade et al. (2013), trabalhado com a identificação de 25 compostos por CLAE em diferentes sucos de uva do vale São Francisco, identificou e quantificou esses compostos em sucos de uvas. Os valores para ácido gálico ficaram entre 1,92 e 5,42 $\mathrm{mg} \mathrm{L}^{-1}$; do ácido cafeico entre 0,14 e 3,90 $\mathrm{mg} \mathrm{L}^{-1}$ e do resveratrol entre 0,05 e 0,67 $\mathrm{mg} \mathrm{L}^{-1}$.

Os coeficientes de correlação calculados para ácido cafeico versus fenóis totais e ácido cafeico versus capacidade antioxidante estão as Figuras 6 e 7. Tanto FT $(r=0,89404)$ como CA $(r=0,91995)$ mantiveram uma correlação forte e positiva com ácido cafeico. ANT versus ácido cafeico também, $r=0,92451$. Tanto o ácido gálico como o resveratrol mostraram correção fraca com $F T$, ANT e CA, $r<0,5$. Minussi et al. (2013), trabalhando com extratos de vinho branco e tinto, encontraram correlação forte para ácido gálico e cafeico, mas baixa para resveratrol.

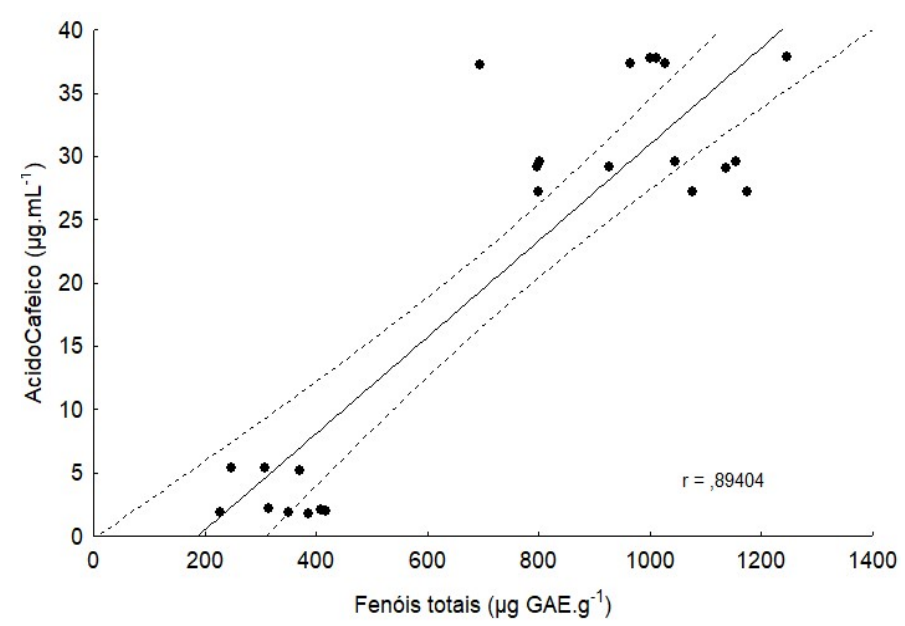

Figura 6: Correlação entre ácido cafeico e fenóis totais.

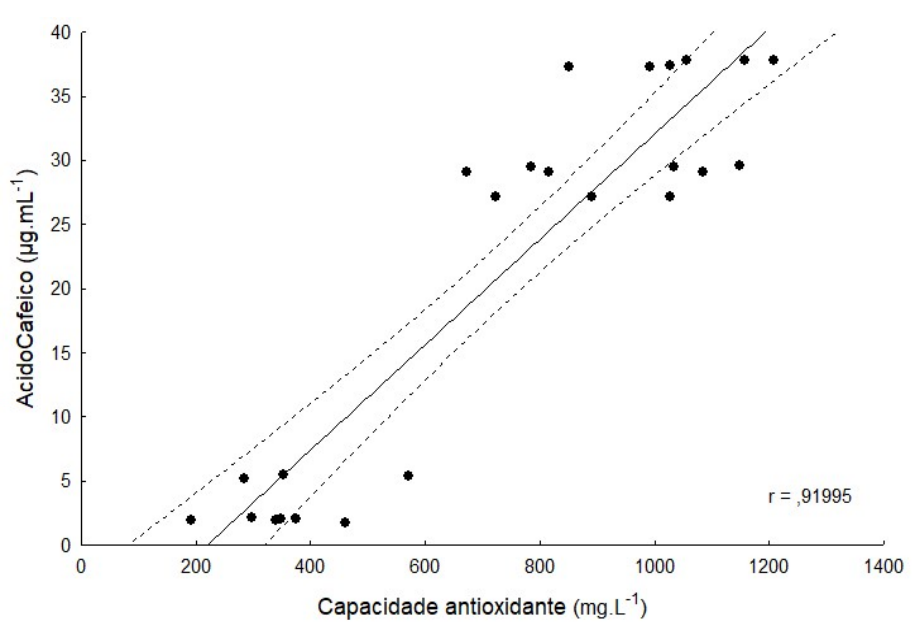

Figura 7: Correlação entre ácido cafeico e capacidade antioxidante.

\section{CONCLUSÕES}

Para os oito tratamentos, em que se estudou o efeito da relação solvente e água na extração de compostos fenólicos e açúcares redutores em resíduo de uva da cultivar Bordô in vitro, destacaram-se positivamente as relações 50 e $75 \%$ v/v de solvente, tanto para metanol como etanol, em relação TFT, TAT, 
AA e AR. Além disso, o metanol puro mostrou-se superior como solvente frente à água pura e o etanol puro, exceção para valor de AR. Já o etanol puro mostrou-se positivo para AR, mas a água pura não. A melhor explicação para esse fato é que parte desse açúcar está na forma glicosilada com as antocianidinas.

Houve alta correlação direta entre a FT, CA e ANT, sendo que dos três compostos identificados por HPLC, o ácido cafeico destacou-se positivamente para atividade antioxidante. O resveratrol teve uma correlação moderada e o ácido gálico a contribuição é inversa. As curvas de calibrações para FT e CA seguiram a Lei de Lambert-Beer, sendo que a relação entre absorção versus FT e absorção versus CA são lineares e crescentes com absortividade molar de $27280 \mathrm{~L} \mathrm{~mol}^{-1} \mathrm{~cm}^{-1}$ e 186 , respectivamente. A curvas de calibração para o ácido gálico, cafeico e resveratrol também tiveram comportamento linear e crescente.

Finalmente, os resultados in vitro indicam que a mistura solvente e água, tanto usando etanol como metanol, na proporção de 50 ou 75 \%, melhora a extração dos compostos fenólicos pelo aumento da solubilidade e, como consequência, facilita a permeabilidade e transferência desses compostos por difusão. Porém, os resultados deste trabalho não são conclusivos em relação à natureza química que foi favorecida frente aos oito tratamentos in vitro, mas os resultados para AR indicam que os compostos fenólicos estão na forma complexa.

\section{REFERÊNCIAS}

ALVES, L. A.; ALMEIDA; SILVA, J. B.; GIULIRTTE, M.. Solubility of D-Glucose in water and ethanol/water mixtures. Journal of Chemical \& Engineering, v.52, p.2166-2170, 2007. DOI: https://doi.org/10.1021/je700177n

ARVANITOYANNIS, I. S.; LADAS, D.; MAVROMATIS.. Potential uses and applications of treated wine waste: a review. International Journal of Food Science and Technology. DOI: https://doi.org/10.1111/j.1365-2621.2005.01111

BABBAR, N.; OBEROI, H. S.; UPPAI, D. S.; PATIL, R. T.. Total phenolic content and antioxidant capacity of extracts obtained from six important fruit residues. Food Research International, v.44, n.1, p.391-396, 2011.DOI:

https://doi.org/10.1016/i.foodres.2010.10.001

BUCIĆ-KOJIĆ, A.; PLANINIĆ, M.; TOMAS, S.; JAKOBEK, L.; SERUGA, M.. Influence of solvent and temperature on extraction of phenolic compounds from grape seed, antioxidant activity and colour of extract. Food Science \& Technology, v.44, p.394-2401, 2009. DOI: https://doi.org/10.1111/j.1365-2621.2008.01876.x

CACACE, J.; MAZZA, G.. Mass transfer process during extraction of phenolic compounds from milled berries. Journal of Food Engineering, v.59, n.4, p.379-389, 2003. DOI: https://doi.org/10.1016/S0260-8774(02)00497-1

CANTILLANO, R. F. F.; ÁVILA, J. M. M.; PERALBA M. C. R.; PIZZOLATO T. M.; TORALLES, R. P.. Actividad antioxidante, compuestos fenólicos y ácido ascórbico de frutillas en dos sistemas de producción. Horticultura Brasileira, v.30, n.4, p. 620-626, 2012. DOI: http://dx.doi.org/10.1590/S0102$\underline{05362012000400010}$

CARMONA, E.; MORENO, M. T.; AVILÉS, M.; ORDOVÁS, J..
Use of grape marc compost as substrate for vegetable seedlings. Scientia Horticulturae, v.137, p.69-74, 2012. DOI: https://doi.org/10.1016/j.scienta.2012.01.023

CAPONE, D. L.; PARDON, K. H.; CORDENTE, A. G.; JEFFERY, D. W.. Identification and quantitation of 3-Scysteinylglycinehexan-1-ol (Cysgly-3-MH) in Sauvignon blanc grape juice by HPLC-MS/MS. Journal of Agricultural and Food Chemistry, v.59, p.11204-11210, 2011. DOI: https://doi.org/10.1021/if202543z

CHENG, V. J.; BEKHIT, A. E. A.; MCCONNELL, M.; MROS, S.; ZHAO, J.. Effect of extraction solvent, waste fraction and grape variety on the antimicrobial and antioxidant activities of extracts from wine residue from cool climate. Food Chemistry, v.134, p.474-482, 2012. DOI: https://doi.org/10.1016/j.foodchem.2012.02.103

COLPO, A. Z. C.; ZAGO, A. C.; SILVA, J. C.; BRAGANÇA, G. C. M. BORTOLONI, V. M. S.. Atividade antioxidante da farinha de couve manteiga e seu efeito nos parâmetros bioquímicos em Drosophila Melanogaster. Brazilian Journal of Health Review, v.2, n.4, p.2796-2801, 2019. DOI: https://doi.org/10.34119/ bjhrv2n4-048

ĆUJIĆ, N.; ŠAVIKIN, K.; JANKOVIĆ, T.; PLJEVLJAKUŠIĆ, D.; ZDUNIĆ, G.; IBRIĆ, S.. Optimization of polyphenols extraction from dried chokeberry using maceration as traditional technique. Food Chemistry, v.194, p.135-142, 2016. DOI: https://doi.org/10.1016/j.foodchem.2015.08.008

FERNANDES, L.; CASAL, S.; CRUZ, R.; PEREIRA, J. A.; RAMALHOSA, E.. Seed oils of ten traditional Portuguese grape varieties with interesting chemical and antioxidant properties. Food Research International, v.50, p.161-166, 
2013. DOI: https://doi.org/10.1016/i.foodres.2012.09.039

FERREIRA, M. V.. Produção de invertase isoladas de levedura de pêssego: Identificação de leveduras, extração otimizada e caracterização cinética das invertases livre e imobilizadas. Dissertação (Mestrado em Ciência e Tecnologia de Alimentos) - Universidade Federal de Pelotas, Pelotas, 2016.

FERRER, J.; PÁEZ, G.; MÁRMOL, Z.; RAMONES, E.; CHANDLER, C.; MARÍN, M.; FERRER, A.. Agronomic use of biotechnologically processed grape wastes. Bioresource Technology, v.70, p.39-44, 2001. DOI: https://doi.org/10.1016/S0960-8524(00)00076-6

GLAMPEDAKI, P.; DUTSCHK, V.. Stability studies of cosmetic emulsions prepared from natural products such as wine, grape seed oil and mastic resin. Colloids and Surfaces. Physicochemical and Engineering Aspects, v.460, p.306-311, 2014. DOI: https://doi.org/10.1016/j.colsurfa.2014.02.048

GONZÁLEZ-CENTENO, M. R.; COMAS-SERRA, F.; FEMNIA, A.; ROSSELLÓ, C.; SIMAL, S.. Effect of power ultrasound application on aqueous extraction of phenolic compounds and antioxidant capacity from grape pomace (Vitis vinifera L.): Experimental kinetics and modeling. Ultrasonics Sonochemistry, v.22, p.506-514, 2015. DOI: https://doi.org/10.1016/j.ultsonch.2014.05.027

GONZÁLEZ-PARAMÁS, A. M.; ESTEBAN-RUANO, S.; SANTOSBUELGA, C.; PASCUAL-TERESA, S.; RIVAS-GONZALO, J. C.. Flavanol Content and Antioxidant Activity in Winery Byproducts. Journal of Agricultural and Food Chemistry, v.52, p.234-238, 2004. DOI: https://doi.org/10.1021/jf0348727

IBGE. Indicadores IBGE: levantamento Sistemático da Produção Agrícola: Rio de Janeiro, 2019. JIMÉNEZ, J. P.; SERRANO, J.; TOBERNETO, M.; ARRANZ, S. M. D.; DÍAZ-RUBIRO, M. E.; GARCÍA-DIZ, L.; GOÑI, I.; SAURACALIXTO, F.. Effects of grape antioxidant dietary fiber in cardiovascular disease risk factors. Nutrition, v.24, p.646653. DOI: https://doi.org/10.1016/i.nut.2008.03.012

JAYAPRAKASHA, G. K.; SINGH, R. P.; \& SAKARIAH, K. K.. Antioxidant activities of grape seed (Vitis vinifera) extracts on peroxidation models in vitro. Food Chemistry, v.73, p. 285-290, 2001. DOI: https://doi.org/10.1016/S03088146(00)00298-3

KAUR, M.; MANDAIR, R.; AGARWAY, R.; AGARWAY, R.. Grape seed extract induces cell cycle arrest and apoptosis in human colon carcinoma cells. Nutrition and Cancer, v.60, p.2-11, DOI: https://doi.org/10.1080/01635580802381295

LEES, D. H.; FRANCIS, F. J.. Standardization of pigment analyses in cranberries. Hort Science, v.7, n.1, p.83-84, 1972. DOI: https://doi.org/10.12691/ jfnr-4-2-3

LOU, S. N.; LIN, Y. S.; HSU, Y. S.; CHIU, E. M.; HO, C. T.. Soluble and insoluble phenolic compounds and antioxidant activity of immature calamondin affected by solvents and heat treatment. Food Chemistry, v.161 p.246-253, 2014. DOI: https://doi.org/10.1016/j.foodchem.2014.04.009
MARTINS, C. R.; LOPES, W. A.; ANDRADE, J. B.. Solubilidade das substâncias orgânicas. Química Nova, v.36, n.8, p. 124J81255, 2013. DOI: https://doi.org/10.1590/S0100$\underline{40422013000800026}$

MAIER, T.; SCHIEBER, A.; KAMMERER, D. R.; CARLE, R.. Residues of grape (Vitis vinifera L.) seed oil production as a valuable source of phenolic antioxidants. Food Chemistry, v. 112, p.551-559, 2009. DOI: https://doi.org/10.1016/i.foodchem.2008.06.005

MELLO, L. M. R.. Vitivinicultura brasileira: panorama 2018, Bento Gonçalves, 2018.

MINUSSI, R. C., ROSSI, M.; BOLOGNA, L.; CORDI, L.; ROTILIO, D; PASTIRE, G. M.; DURÁN, N.. Phenolic compounds and total antioxidant potential of commercial wines. Food Chemistry, v.82, p.409-416, 2003. DOI: https://doi.org/10.1590/fst.07818

NATIVIDADE, M. M. P.; CORRÊA, L. C; SOUZA, S. V. C.; PEREIRA, G. E.; LIMA, C. O.. Simultaneous analysis of 25 phenolic compounds in grape juice for HPLC: Method validation and characterization of São Francisco Valley samples. Microchemical Journal, v.110, p.665-674, 2013. DOI: https://doi.org/10.1016/j.microc.2013.08.010

NEGRO, C., TOMMASI, L., MICELI, A.. Phenolic compounds and antioxidant activity from red grape marc extracts. Bioresource Technology, v.87, p.41-44, 2003.DOI: https://doi.org/10.1016/S0960-8524(02)00202-X

OZGEN, M., REESE, R. N.; TULIO, A. Z.; SCHEERENS, J. C.; MILLER, A. R.. Modified 2,2-azinobis- 3-ethylbonzothiazoline6-sulfonic acid (ABTS) method to measure antioxidant capacity of selected small fruits and comparison to ferric reducing antioxidant power (FRAP) and 2,2'-diphenyl-1picrylhydrazyl (DPPH) methods. Journal of Agricultural. Food Chemistry, v.54, p.1151-1157, 2006. DOI: https://doi.org/10.1021/jf051960d

PASKO, P.; BARTÓN, H.; ZAGRODZKI, P.; GORINSTEIN, S.; ZACHWIEJA, Z.. Anthocyanins, total polyphenols and antioxidant activity in amaranth and quinoa seeds and sprouts during their growth. Food Chemistry, v.115, n.3, p. 994-998, 2009. DOI: https://doi.org/10.1016/j.foodchem.2009.01.037

RIBEIRO, L. F.. Avaliação dos compostos bioativos e atividade antioxidante in vitro e in vivo em bagaços de uvas (Vitis vinífera e VItis labrusca). Tese (Doutorado em Engenharia de Alimentos) - Universidade Federal do Paraná, 2016.

ROCKENBACK, I. I.; RODRIGUES, E.; GONZAGA, L. V.; CALIARI, V.; GENOVESE, M. I.; GONÇALVES, E. S. S.; FETT, R.. Phenolic compounds content and antioxidant activity in pomace from selected red grapes (Vitis vinifera L. and Vitis labrusca L.) widely produced in Brazil. Food Chemistry v.127, n.1, p.174179, 2011. DOI:

https://doi.org/10.1016/j.foodchem.2010.12.137 
RODRÍGUEZ-JIMENES, G. C.; ARMANDO, V. G; ESINOZAPÉREZ, D. J.; SALGADO-CERVANTES, M. A.; ROBLES-OLVERA, V. J.; GARCIA-ALVARADO, M. A.. Mass Transfer during Vanilla Pods Solid-Liquid Extraction: effect of Extraction Method. Food Bioprocess Technology, v.6, n.10, p.2640-2650, 2013. DOI: https://doi.org/10.1007/s11947-012-0975-6

SAHPAZIDOU, D.; GEROMICHALOS, G. D.; STAGOS, D.; APOSTOLOU, A.; HAROUTOUNIAN, S. A.; TSATSAKIS, A. M.; TZANAKAKIS, G. N.; HAYES, A. W.; KOURETAS, D.. Anticarcinogenic activity of polyphenolic extracts from grape stems against breast, colon, renal and thyroid cancer cells. Toxicology Letters, v.230, p.218-224, 2014. DOI: https://doi.org/10.1021/jf8024979

SHROTRIYA, S.; TYAGI, A.; DEEP, G.; ORLICKY, D. J.; WISELL, J.; WANG, X-J; SCLAFANI, R. A.; AGARWAL, R.; AGARWAL, C.. Grape Seed Extract and Resveratrol Prevent 4-Nitroquinoline 1-Oxide Induced Oral Tumorigenesis in Mice by Modulating AMPK Activation and Associated Biological Responses. Molecular Carcinogenesis, v.54, p.291-300, 2015.DOI: https://doi.org/10.1002/mc.22099

SOUZA, V. B.; FUJITA, A.; THOMAZINI, M.; SILVA, E. R.; LUCON, J. F.; GENOVESE, M. F.; FAVARO-TRINDADE, C. S.. Functional properties and stability of spray-dried pigments from Bordo grape (Vitis labrusca) winemaking pomace. Food Chemistry, v.164, p.380-386, 2014. DOI: https://doi.org/10.1016/j.foodchem.2014.05.049

SILVA, R. S.; VENDRUSCOLO, J. L.; TORALLES R. P.. Avaliação da capacidade antioxidante em frutas produzidas na região sul do RS. Revista Brasileira Agrociência, v.17, n.3-4, p.392400, 2011.
SINGLETON, V. L.; ROSSI, J. A.. Colorimetry of total phenolics with phosphomolybidic-phosphotungstic acid reagents. American Journal of Enologie and Viticulture, v.16, p.144158, 1965

STALMACH, A.; EDWARDS, C. A.; WIGHTMAN, J. D.; CROZIER, A.. Identification of (poly) phenolic compounds in concord grape juice and their metabolites in human plasma and urine after juice consumption. Journal of Agricultural and Food Chemistry, v.59, n.17, p.9512-9522, 2011. DOI: https://doi.org/10.1021/if2015039

VENKATESAN, T.; CHOI, Y. W.; KIM, Y. K.. Impact of different extraction solvents on phenolic content and antioxidant potential of Pinus densiflora back extract. BioMed Hindawi. 2019. DOI: https://doi.org/10.1155/2019/3520675

VERGARA-SALINAS, J. R.; VERGARA, M.; ALTAMIRANO, C.; GONZALEZ, A.; PEREZ-CORREA, J. R.. Characterization of pressurized hot water extracts of grape pomace: chemical and biological antioxidant activity. Food Chemistry, v.171, p.62-69, 2015. DOI:

https://doi.org/10.1016/i.foodchem.2014.08.094

$\mathrm{XU}$, B.; CHANG, S. K. C.. Total Phenolic Acid, Anthocyanin, Flavan-3-ol, and Flavonol Profiles and Antioxidant Properties of Pinto and Black Beans (Phaseolus vulgaris L.) as Affected by termal Processing. Journal Agricultural and Food Chemistry, v.57, p.4754-4764. DOI: https://doi.org/10.1021/jf900695s

ZHU, F.; DU, B.; ZHENG, L.; LI, J.. Advance on the bioactivity and potential applications of dietary fibre from grape pomace. Food Chemistry, v.186, p.207-212, 2015. DOI: https://doi.org/10.1016/j.foodchem.2014.07.057

A CBPC - Companhia Brasileira de Produção Científica (CNPJ: 11.221.422/0001-03) detém os direitos materiais desta publicação. Os direitos referem-se à publicação do trabalho em qualquer parte do mundo, incluindo os direitos às renovações, expansões e disseminações da contribuição, bem como outros direitos subsidiários. Todos os trabalhos publicados eletronicamente poderão posteriormente ser publicados em coletâneas impressas sob coordenação da Sustenere Publishing, da Companhia Brasileira de Produção Científica e seus parceiros autorizados. Os (as) autores (as) preservam os direitos autorais, mas não têm permissão para a publicação da contribuição em outro meio, impresso ou digital, em português ou em tradução. 